\title{
Dose Use of Sauna Fit for All Individual?
}

\author{
Nasim Habibzadeh ${ }^{1 *}$ and Daniel Wood $^{2}$ \\ ${ }^{1}$ Department of Sport Science, Teesside University, UK \\ ${ }^{2}$ BSc Student in Sport Therapy and Rehabilitations, UK \\ *Corresponding Author: Nasim Habibzadeh, Department of Sport Science, Teesside University, UK. \\ E-mail: nasimhabibzadeh@yahoo.com
}

Received: September 16, 2019; Published: September 26, 2019

DOI: $10.31080 /$ ASMS.2019.03.0421

\begin{abstract}
Fatigue and tiredness can occur in competitive sports or in any other physical movements. Fatigue often is along with depletions in both physical and mental powers. Prolonged fatigues can cause to the illnesses or injuries. Following various types of fatigues after any exercise endeavourer individuals attempt to recover their exhaustions and to relax for another effort. Sauna improves circulations, immune system and join movements but at the same time can lead to the loss of blood flow and can cause dehydration in human body. Owing to this, sauna is a good approach for athletes to relief their fatigues but it is not recommended for all .The sportspersons normally drink great deals of fluids and usually know how to use sauna or when is required to use it. Therefore, for non-athletes individual cautions are needed to be considered when they are intending to use sauna bathing otherwise they can be at risk of serious health conditions.
\end{abstract}

Keywords: Fatigue; Sauna; Benefits; Side - Affects

\section{Introduction}

The onset of fatigue normally occurs during any ordinary movements or sport performances which simply may block performances [1]. Physiologists usually describe fatigue as acute capacity - induced decline in variety of physical tasks [2] which is associated with both physical and cognitive consequences [3].

More specifically, fatigue is a physiological impairments condition with feeling of lack of energy and exhaustion owing to the decreases in metabolic, hormonal, neural functions during specific movement task $[4,5]$. In this condition, muscles blood flow and oxygen uptake $\left(\mathrm{O}_{2}\right)$ and ATP (Adenosine triphosphate) decline and muscles are not able to contract optimally in longer times [6]. Correspondingly, prolonged physical fatigue can lead to the mental fatigue by inducing energy depletion in brain energy which limits physical performances and causes decline in abilities [7].

The onset of fatigue which results in decrements in capabilities often occurs in different movements. Although ,the degree of tiredness and inability depending of the hardship - of - related activity is vary in different movement task [8]. Long - term fatigues can lead to the illnesses or injuries in any given task during any physical efforts in clinical terms [9]. Even though, the type of fatigues are various during different movement which can cause differences in pain and injuries in different individuals [10].

The symptoms of fatigues are more remarkable in sports such as track and field and swimming. Endurance runners or swimmers normally have high level of physical and mental capacities in various sports events. Nonetheless, there should be the times at which they could restore their consumed energy and lost power for another match or competition. Rebuilding the energy levels after any tournament assists to recover the exhaustions and prevent chronic fatigues $[11,12]$.

Basketball, handball, volleyball, rugby, soccer and many other similar worldwide sports players have to compete in various rounds during different games in a match. The levels of stamina and power in these types of sport are significantly important although always there are some acute fatigues in sportspersons after desperate games [13-15].

Following any kind of fatigues owing to any hard efforts, individuals attempt to improve their physical and mental exhaustions and impairments in various ways such as consuming good nutrients, massage and taking some rest for some periods of time for another competitive endeavourer [16]. 
Likewise, some people use sauna to recover their tiredness and to relax. Sauna helps to increase better blood flow in musculature which improves muscles soreness and joint movements. Sweating during sauna leads to relief from produced toxic material induced from muscle damage during hard any efforts. Sweating in sauna is kind of therapy for athletes since decreases aforementioned stress and reinforced the immune system in body in part in elites compared to untrained individuals $[17,18]$.

Nonetheless, prolonged utilizations of Sauna bathing enhance the risk of dehydration and fluid loss in human body which can cause to the low blood pressures and in its worse condition to loss of consciousness in ordinary people in particular [19]. Although hot sauna bathing usually increase circulation in different individual like athlete in sport fields or patients with heart failure syndromes but over-sweating in hot sauna bathing normally declines the body water and blood flow in a long-term usage in a session. Therefore, replacing sufficient fluid substitutes is a necessary task for all type of groups either athletes or non-athletes peoples [20].

Further, some individual use sauna hot bathing to reduce their obesity as they assume over - sweating in sauna stream room , decreases body fat rates and consequently they can simply lose weight. With regards to this, some athletes in some points in their sporting life require to reduce their body weight and sauna bathing is a good strategy for them to change their body mass index in quicker times. This kind of elites individual as they have high level of fitness, for them losing weight with this approach is not too risky but for ordinary people (i.e. obese individual) who never used sauna bathing before, it can be dramatically dangerous to lose weight in this way owing to the dehydration problems. Performing some gentle exercises such as a simple walking for sedentary individual can be more beneficial technique to lose weight and to stay healthy [21].

\section{Conclusions}

Fatigue can occurs in any physical activity and in part in competitive sporting performances. Different individuals use different strategies to reduce their tiredness and exhaustions . Among different approaches, sauna bathing is kind of implication which utilizes often by athletes in different sport fields for various purposes. Athletes' use sauna bathing to relief their acute or chronic fatigues and more specifically use it for detoxation that caused by muscle damage during sport ' workouts. They also use hot sauna for losing weight programs in technical ways. Utilizing sauna is normally is along with dehydrators and fluid loss in human body. Athletes individual usually know how to use the sauna bathing to gain the most benefit of it but ordinary individuals who nerve use sauna bathing before are require to have full knowledge whenever intend to use it . Replacing sufficient water and fluids is dramatically vital after sauna bathing otherwise individual can be at risk of serious health problems.

\section{Bibliography}

1. Monte A Dal. "Fatigue and sport". Functional Neurology 17 (2002): 7-10.

2. Enoka RM and Duchateau J. "Muscle fatigue: what, why and how it influences muscle function". Journal of Physiology 5 (2008): 11-23.

3. Lorist MM., et al. "Motor fatigue and cognitive task performance in humans". Journal of Physiology 545 (2002): 313-319.

4. Puetz TW. "Physical activity and feelings of energy and fatigue: epidemiological evidence". Sports Medicine 36 (2006): $767-$ 780.

5. Allen D., et al. "Skeletal muscle fatigue: cellular mechanisms". Physiology Review 88 (2008): 287-332.

6. Wan JJ., et al. "Muscle fatigue: general understanding and treatment". Experimental and Molecular Medicine 49 (2017): e384.

7. McMorris T., et al. "Cognitive fatigue effects on physical performance: A systematic review and meta-analysis". Physiology Behavior 188 (2018): 103-107.

8. Wentzell M., et al. "Fatigue in Sport and Exercise". The Journal of the Canadian Chiropractic Association 60 (2016): 125.

9. Jones CM., et al. "Training Load and Fatigue Marker Associations with Injury and Illness: A Systematic Review of Longitudinal Studies". Sports Medicine 47 (2017): 943-974.

10. Stiles PG. "Types of fatigue". American Journal of Public Health 10 (1920): 653-656.

11. Goodall., et al. "Neuromuscular fatigability during repeatedsprint exercise in male athletes". Medicine and Science in Sports and Exercise 47 (2015): 528-536.

12. Brown $\mathrm{S}$ and Kilding AE. "Exercise-induced inspiratory muscle fatigue during swimming: the effect of race distance". The Journal of Strength and Conditioning Research 25 (2011): 12041209.

13. Rampinini E., et al. "Match-related fatigue in soccer players". Medicine and Science in Sports and Exercise 43 (2011): 21612170.

14. Coutts A., et al. "Changes in selected biochemical, muscular strength, power, and endurance measures during deliberate overreaching and tapering in rugby league players". International Journal of Sports Medicine 28 (2007): 116-124.

15. Montgomery PG., et al. "Muscle damage, inflammation, and recovery interventions during a 3-day basketball tournament". European Journal of Sport Science 8 (2008): 241-250. 
16. Carroll TJ., et al. "Recovery of central and peripheral neuromuscular fatigue after exercise". Journal of Applied Physiology 122 (2017): 1068-1076.

17. Crinnion WJ. "Sauna as a valuable clinical tool for cardiovascular, autoimmune, toxicant-induced and other chronic health problems". Alternative Medicine Review 16 (2011): 215-225.

18. Pilch W., et al. "Effect of a Single Finnish Sauna Session on White Blood Cell Profile and Cortisol Levels in Athletes and Non-Athletes". Journal of Human Kinetics 39 (2013): 127-135.

19. "Sauna: Health benefits, risks, and precautions". Medical News Today.

20. Hannuksela ML and Ellahham S. "Benefits and risks of sauna bathing". The American Journal of Medicine 110 (2001): 118126.

21. Infrared Sauna for Weight Control and Obesity | Good Health Saunas. Infrared Sauna Health Benefits.

Volume 3 Issue 10 October 2019

\section{(C) All rights are reserved by Nasim Habibzadeh and} Daniel Wood. 